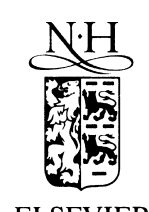

Nuclear Instruments and Methods in Physics Research B 153 (1999) 270-274

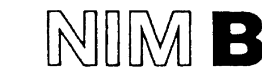

Beam Interactions

with Materials \& Atoms

ELSEVIER

\title{
Molecular Dynamics simulation of the desorption of molecules by energetic fullerene impacts on graphite and diamond surfaces
}

\author{
M. Kerford *, R.P. Webb \\ SCRIBA, School of Electronic Engineering, Information Technology and Mathematics, University of Surrey, Guildford, Surrey, GU2 \\ $5 X H, U K$
}

\begin{abstract}
Molecular Dynamics computer simulations have been used to investigate the energy propagation following an energetic fullerene impact on both diamond and graphite lattices. Transverse and longitudinal waves are seen to propagate across the surface particularly for the graphite. This paper investigates the possibility of desorption of large molecules - both physisorbed and chemisorbed - from the surface due to the passage of these waves. SIMS experiments using cluster ions suggest that this could be a viable technique for desorbing large intact molecules from surfaces. So far SIMS work has concentrated on the use of metal surfaces to enhance the secondary ion emission. We investigate here the use of carbon targets - in particular graphite - where the possibility of ejection of large intact molecules is potentially greater. (c) 1999 Elsevier Science B.V. All rights reserved.
\end{abstract}

\section{Introduction}

Secondary Ion Mass Spectrometry (SIMS) has traditionally been performed using high energy monomer particles, which cause damage to the surface of the sample. Recent work has been looking at the use of complex (cluster or molecular) projectiles as an alternative to the monomer projectiles currently used [1]. Experimental work has shown an enhancement occurs in the secondary ion yield with increasingly complex projectiles [2]. Simulation studies have looked at the ejection of small molecules from surfaces as a function of the adsorbate size [3] and as a function of the

\footnotetext{
${ }^{*}$ Corresponding author. Tel.: +44 1483300 800; fax: +44 1483534 139; e-mail: m.kerford@ee.surrey.ac.uk
}

cluster size for small clusters [4]. Simulations have also been performed to look at the fragmentation pattern of sputtered molecules [5]. Both simulation and experimental work has concentrated on metallic surfaces. This is because the secondary ion emission from metals will be high.

The reason for using cluster beams is to try and desorb large molecules from a surface. With single atomic ions the cascade induced in the surface region is often not sufficiently dense to create the necessary conditions to desorb a large molecule intact [3]. To desorb a large intact molecule cascading target particles must strike the adsorbed molecule simultaneously in a similar direction to impart enough energy to the molecule to lift it from the surface.

In the work presented here we look at the effect of surface acoustic waves seen to propagate from 
the impact of clusters on a graphite surface [6]. We compare the effects of impact on a graphite surface with that of a diamond surface where the amplitude of such acoustic waves are not as large [6].

\section{The simulation method}

The Molecular Dynamics simulation model used in this study has been described in detail in other publications [7,8] and so only a brief description will be given here. The program uses the Brenner many-body potentials [9] to model the $\mathrm{C}-\mathrm{C}$ and $\mathrm{C}-\mathrm{H}$ interactions. These potentials are known to model graphite and diamond lattices well for the systems used here, as well as giving stable hydrocarbon molecules of the type used. However, the cut-off distance of this potential is such that there is no inter-planar bonding between the graphite layers. It is possible to introduce such a long range term to add more realism to these simulations, but the increase in the needed computer time to calculate these longer range potentials limits there usefulness at the present. Work using such a potential indicates that the acoustic waves seen in these simulation studies still occur with similar magnitude and duration [10]. The potential gives the correct mechanical properties for both graphite and diamond.

Two targets are used in this study, graphite and diamond. The graphite target consists of 13 atomic layers, each of dimension $100 \AA$ by $100 \AA$. This contains approximately 50000 atoms. The initial temperature of the lattice is $0 \mathrm{~K}$. The diamond lattice consists of approximately 74000 atoms with dimensions of $100 \AA$ by $100 \AA$ by $40 \AA$, again with initial temperature of $0 \mathrm{~K}$. Free boundaries are used to avoid reflection of energy back into the lattice from its edges. Correct treatment at simulation boundaries is always difficult and we adopt the policy of keeping the boundaries as far away from the area of interest as possible. This is achieved by using a large crystallite. The main results of this work occur before much of the energy has reached the lattice boundaries. The longitudinal wave hits the boundaries early in the simulation but it propagates only a very small amount of energy by comparison with the slower moving transverse wave.

Twelve $\mathrm{C}_{6} \mathrm{H}_{5}$ molecules are chemisorbed to the surface of the lattice, placed directly above an atom as this provides the strongest binding. For the graphite lattice this gives a bond strength of about $1.8 \mathrm{eV}$. For the diamond the strength is about $2 \mathrm{eV}$. The $\mathrm{C}_{6} \mathrm{H}_{5}$ molecule is constructed as a benzene ring with one of the hydrogens removed to provide a strong bond to the carbon lattice.

In a separate simulation we physisorbed twelve $\mathrm{C}_{6} \mathrm{H}_{5}$ molecules to the same surfaces but at a distance far enough away so that they are not bound.

We have chosen - rather arbitrarily - to stand the molecules up on the surface, this is so that we can watch for co-ordinated ejection from the surface of these adsorbed molecules.

The simulation solves the equations of motion simultaneously for each particle and stores information on position, velocity and energy states at different timesteps. From the kinetic energies of the particles it is possible to calculate a mean square velocity of the simulated target region. From this one could be tempted to infer a mean temperature for the region. This has been done successfully in the past for metal cluster on metal substrates [11]. In the first of these, Betz et al. [11] showed that a small region of the target arrives at thermal dynamic equilibrium within the first few $100 \mathrm{fs}$ after the impact and the velocity distributions of the particles within the region for a Maxwell-Boltzmann curve. In the second, Colla et al. [12] used a method of gliding averages to describe local temperature variations even when thermal dynamic equilibrium had not been reached for the complete region. As we are interested in the propagation of energy through the target volume we will adopt the latter scheme for this study. Fig. 1 shows the velocity distributions for the whole simulated target volume at different times after impact of a $4 \mathrm{keV} \mathrm{C}_{60}$ molecule. It is clear that the target does not reach a constant temperature until several picoseconds into the simulation. In fact the velocity distributions show that quite quickly the energy of the impacting molecule spreads out but there is a substantial range of temperatures in the simulated region even at quite long times. We therefore follow Colla et al. 


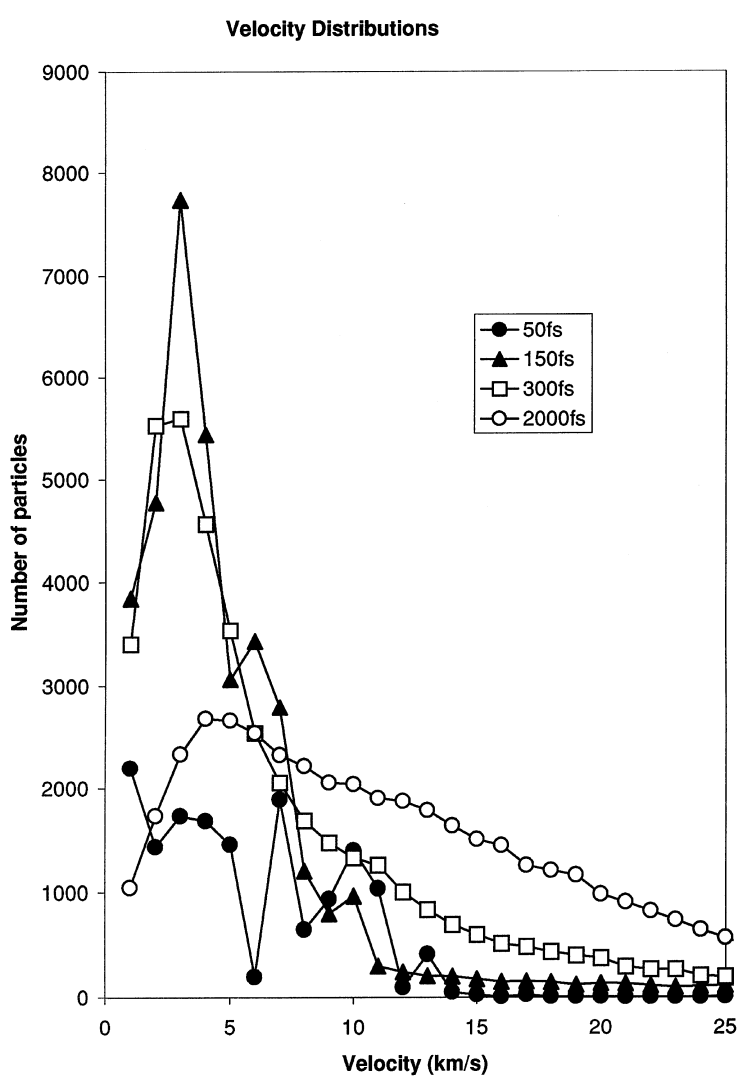

Fig. 1. Velocity distributions of a graphite target as a function of time after a $4 \mathrm{keV} \mathrm{C}_{60}$ impact.

[12] and take a local volume around each particle in the simulation (a $5 \AA$ radius was chosen, although increasing this has little effect on the results presented here). The mean square velocity of the particles inside this volume is then calculated. This enables us to visualise the energy spreading through the target as a function of time.

\section{Results and discussion}

Simulations have been run for $\mathrm{C}_{60}$ impacts with a variety of energies on both graphite and diamond targets. Each simulation has been analysed according to the methods described earlier. Fig. 2 shows the impact of a $\mathrm{C}_{60}$ molecule on a graphite upon which twelve $\mathrm{C}_{6} \mathrm{H}_{5}$ molecules have been positioned. These molecules $-\mathrm{a}$ benzene ring with

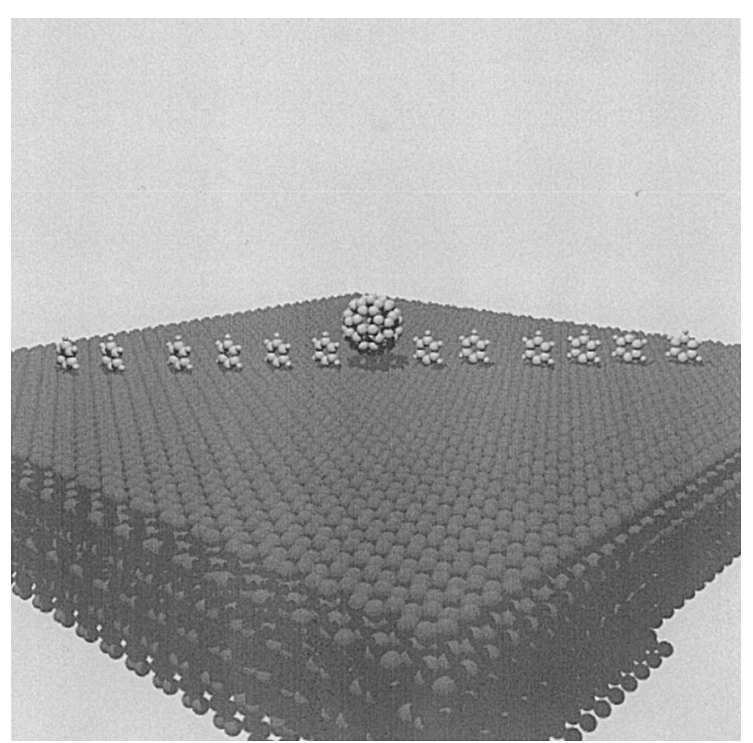

Fig. 2. A graphite surface with 12 adsorbed $\mathrm{C}_{6} \mathrm{H}_{5}$ molecules.

one hydrogen removed - are placed directly above atoms in the graphite target to form a diagonal line across the lattice. They are $1.6 \AA$ above the graphite surface layer, which gives the correct carbon-carbon bond energy for chemisorption to the surface. The $\mathrm{C}_{6} \mathrm{H}_{5}$ molecules are placed at a distance of $1.5 \AA$ in the case of the diamond lattice.

In Fig. 3 pictures of the surface and molecules are shown for different times after the impact. We only show the surface layer to make things clearer and we are looking parallel to the surface plane. As the $\mathrm{C}_{60}$ impacts on the surface energy is spread radially outwards from the impact site, passing energy to the $\mathrm{C}_{6} \mathrm{H}_{5}$ molecules. On the graphite surface a transverse acoustic wave can be seen travelling across the surface and a hillock forms around the impact site. As the surface of the lattice rises and falls with the passing of the transverse acoustic wave the $\mathrm{C}_{6} \mathrm{H}_{5}$ molecules move also. However, insufficient energy is transferred from the wave as it passes a $\mathrm{C}_{6} \mathrm{H}_{5}$ molecule to desorb them. Hence they remain strongly bonded to the surface. One molecule is ejected intact from the graphite surface and a couple of broken molecules are ejected from the impact region on the diamond lattice. Unlike the graphite, diamond exhibits no physical hillock like effects around the impact site 


\section{0keV $\mathrm{C}_{60}$ Impacts}

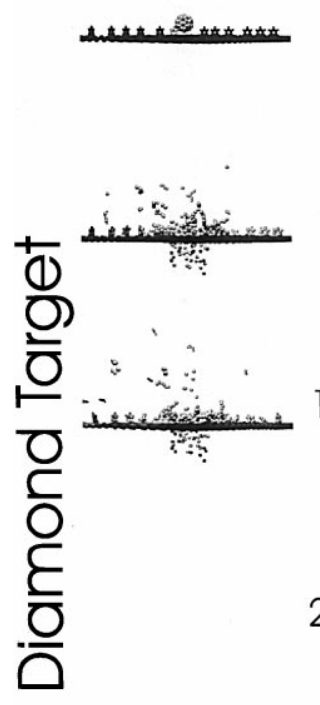

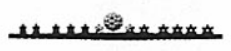

1000fs

500 fs

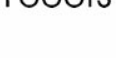

2000fs

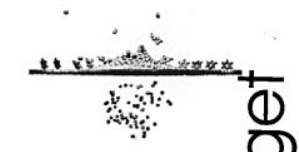

\section{(1)}

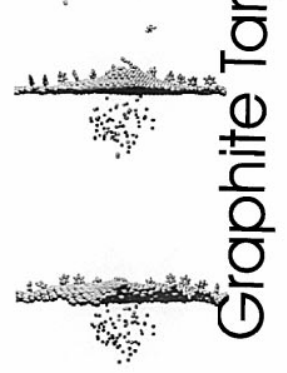

\section{Chemisorbed}

Fig. 3. A sequence of images from a $10 \mathrm{keV} \mathrm{C}_{60}$ impact on diamond and graphite surfaces with twelve $\mathrm{C}_{6} \mathrm{H}_{5}$ molecules chemisorbed to the surface.

and no acoustic wave has been observed. These features are only observed in the graphite due to the planar nature of the lattice. There is therefore no discernible co-ordinated mechanism for transferring energy from the diamond lattice to the $\mathrm{C}_{6} \mathrm{H}_{5}$ other than through direct collision and thus they remain close to their original locations, as can be seen.

Although there is not enough energy passed to a $\mathrm{C}_{6} \mathrm{H}_{5}$ molecule from the transverse acoustic wave created by a $10 \mathrm{keV} \mathrm{C}_{60}$ impact to break the bond, it is possible that a higher energy or greater energy density may cause enough energy to be transferred from the wave to desorb the molecule. Such a cascade can be caused by a larger cluster. This will be examined in future work.

Fig. 4 shows a similar simulation as Fig. 3, however, the height of the $\mathrm{C}_{6} \mathrm{H}_{5}$ molecules above the surface has been increased to $2.7 \AA$ in the case of graphite and $2.0 \AA$ in the case of diamond. This

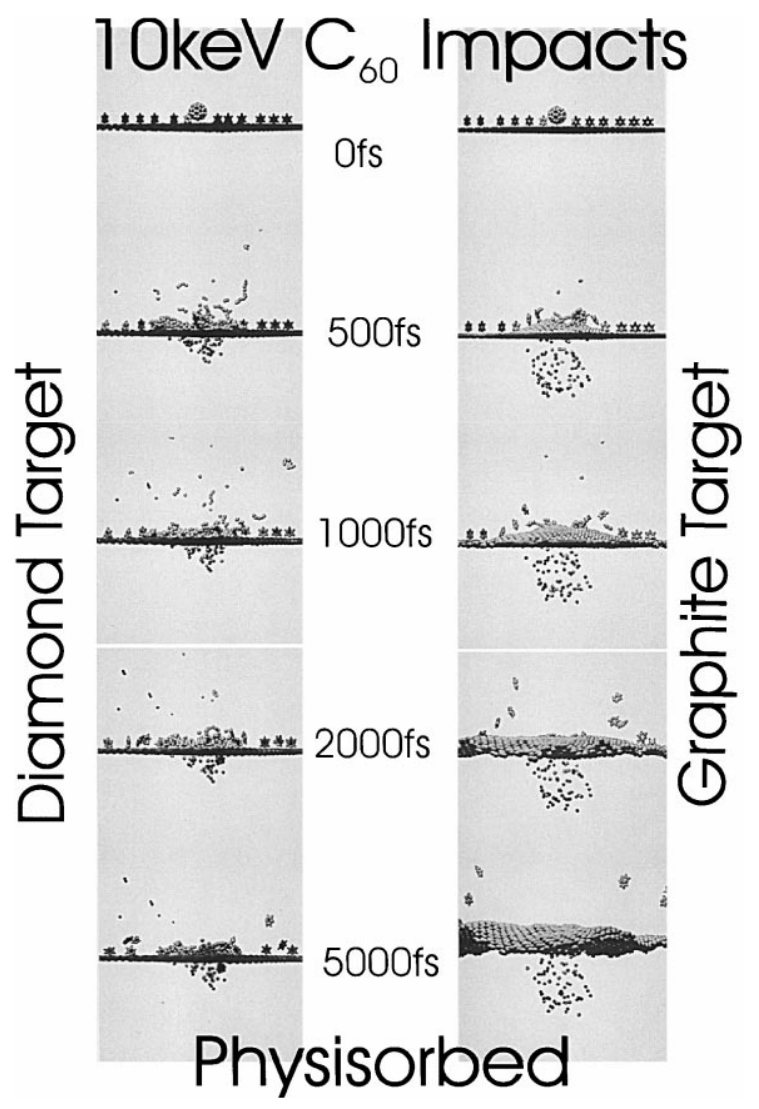

Fig. 4. A sequence of images from a $10 \mathrm{keV} \mathrm{C}_{60}$ impact on diamond and graphite surfaces with twelve $\mathrm{C}_{6} \mathrm{H}_{5}$ molecules physisorbed to the surface.

distance is between the cutoff distance of the potential function and the interlayer separation of the graphite and diamond planes, thus there is no physical bond between the $\mathrm{C}_{6} \mathrm{H}_{5}$ and the surface. Once again a $10 \mathrm{keV}$ impact of a $\mathrm{C}_{60}$ molecule is simulated and is shown in Fig. 4. As the transverse acoustic wave reaches the $\mathrm{C}_{6} \mathrm{H}_{5}$ molecules it passes energy to them.

Fig. 5 shows the potential energy of the molecule as a function of the distance from the surface. It shows a minimum when the carbon atom is $1.6 \AA$ from the surface, which is consistent with the $\mathrm{C}-\mathrm{C}$ bond energy, as used in the simulations shown in Fig. 3. As mentioned earlier, the distance used in the second simulation detailed is $2.7 \AA$, which lies on the plateau of the potential energy curve. The graphite surface moves closer to a $\mathrm{C}_{6} \mathrm{H}_{5}$ molecule 
Potential Energies of Adsorbate Atoms

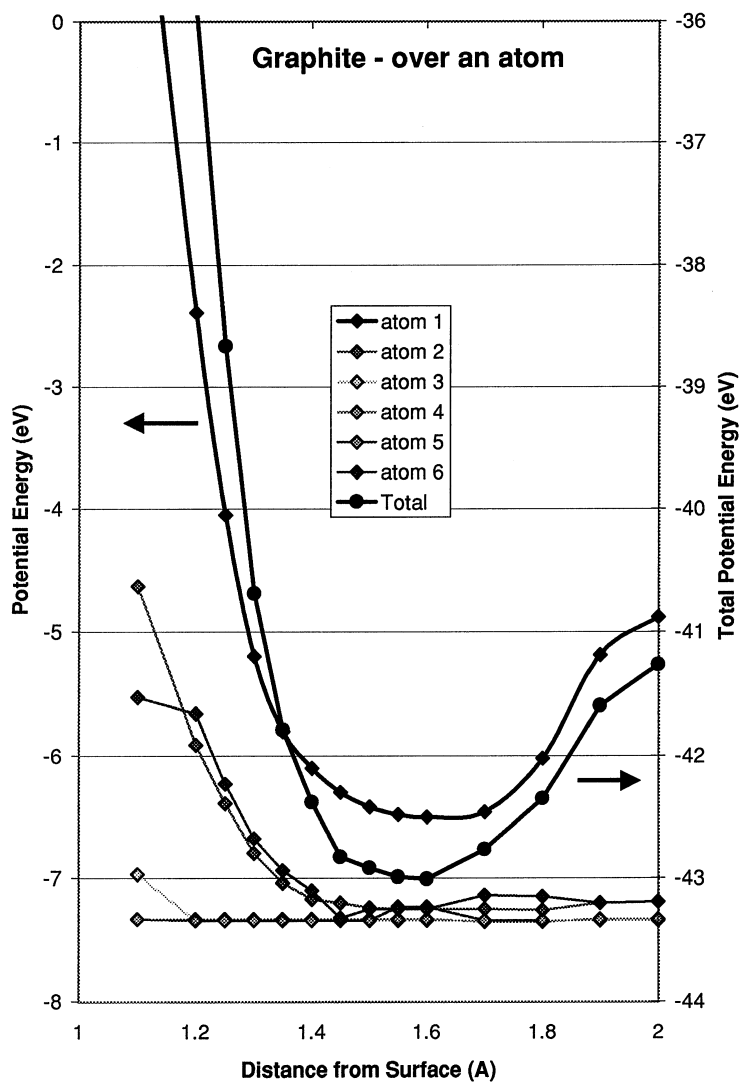

Fig. 5. Potential energies of the atoms of an adsorbed molecule on a graphite surface as a function of the distance from the surface.

as the wave passes, thus the $\mathrm{C}-\mathrm{C}$ separation is reduced. As this separation falls down towards the minimum of the potential energy curve it would be reasonable to assume that a $\mathrm{C}_{6} \mathrm{H}_{5}$ molecule would become bonded to the surface. However, as can be see in Fig. 4 this is not the case. As the wave passes the $\mathrm{C}_{6} \mathrm{H}_{5}$ molecules are repelled from the surface. The interesting thing here is that they are almost peeled from the surface in a co-ordinated fashion. One could easily imagine a large molecule being removed intact in this way by this acoustic wave. Conversely the physisorbed particles on the diamond lattice largely remain on the surface, some of them are desorbed after long times but not in a co-ordinated fashion. This is not surprising as the energy propagation through the diamond lattice does not happen in such a co-ordinated way as in the graphite system.

Further work is underway to investigate this phenomenon more thoroughly and with greater realism.

\section{References}

[1] E.A. Schweikert, M.G. Blain, M.A. Park, E.F. Da Silveira, Nucl. Instr. and Meth. B 50 (1990) 937.

[2] M.G. Blain, S. Della-Negra, H. Joret, Y. LeBeyec, E.A. Schweikert, Phys. Rev. Lett. 63 (15) (1989) 1625.

[3] R.S. Taylor, B.J. Garrison, Chem. Phys. Lett. 230 (1994) 495.

[4] R. Zaric, B. Pearson, K.D. Krantzman, B.J. Garrison, Int. J. Mass Spec. and Ion Proc. 174 (1998) 155.

[5] R. Taylor, B.J. Garrison, Langmuir 11 (1995) 1220.

[6] M. Kerford, R.P. Webb, EMRS Proc, 1998, in press.

[7] D.E. Harrison Jr., Crit. Rev. in Solid State Mat. Sci. 14 (1988) S1.

[8] R. Smith, D.E. Harrison Jr., B.J. Garrison, Phys. Rev. B 40 (1989) 93.

[9] D.W. Brenner, Phys. Rev. B 46 (1992) 1948.

[10] K. Beardmore, R. Smith (private communication).

[11] G. Betz, W. Husinsky, Nucl. Instr. and Meth. B 122 (1997) 311.

[12] Th.J. Colla, H.M. Urbassek, Rad. Eff. and Defects in Solids 142 (1997) 439. 